\title{
Optimal Cycles for Low-Dissipation Heat Engines
}

\author{
Paolo Abiuso®* \\ ICFO_Institut de Ciències Fotòniques, The Barcelona Institute of Science and Technology,08860 Castelldefels (Barcelona), Spain \\ Martí Perarnau-Llobet@ \\ Max-Planck-Institut für Quantenoptik, D-85748 Garching, Germany
}

(Received 21 July 2019; revised manuscript received 31 October 2019; accepted 3 March 2020; published 19 March 2020)

\begin{abstract}
We consider the optimization of a finite-time Carnot engine characterized by small dissipations. We bound the power with a simple inequality and show that the optimal strategy is to perform small cycles around a given working point, which can be, thus, chosen optimally. Remarkably, this optimal point is independent of the figure of merit combining power and efficiency that is being maximized. Furthermore, for a general class of dissipative dynamics the maximal power output becomes proportional to the heat capacity of the working substance. Since the heat capacity can scale supraextensively with the number of constituents of the engine, this enables us to design optimal many-body Carnot engines reaching maximum efficiency at finite power per constituent in the thermodynamic limit.
\end{abstract}

DOI: 10.1103/PhysRevLett.124.110606

The Carnot engine has a pivotal role in thermodynamics, both from a fundamental and applied perspective, being the reference point for other engines in terms of efficiency $[1,2]$. Thus, it is of paramount importance to understand its limits and strategies for its best utilization. In this Letter, we consider the optimization of a finite-time Carnot cycle within the so called low-dissipation (LD) regime [3-14], where the driving of the control parameters is slow but finite. Previous studies of Carnot engines in the LD regime have considered bounds on the reachable efficiencies [3], tradeoffs between efficiency and power [7-9,15], the coefficient of performance of refrigerators $[12,13]$, the impact of the spectral density of the thermal baths [14], and other thermodynamic figures of merit [10,11]. Despite this remarkable progress, the following crucial question has remained unaddressed: given a certain level of control on the working substance (e.g., some parameters of the Hamiltonian, or some macroscopic variables such as volume or pressure), what is the optimal cyclic modulation of the control parameters to maximise the power output (or, more generally, any figure of merit involving power and efficiency $[7-9,15])$ of a finite-time Carnot engine? Such an optimal cycle has been designed for a single-qubit engine in [16,17], but a general understanding is lacking.

Using recent insights on a geometrical approach to thermodynamics [18-29], we show that, given any

Published by the American Physical Society under the terms of the Creative Commons Attribution 4.0 International license. Further distribution of this work must maintain attribution to the author(s) and the published article's title, journal citation, and DOI. Open access publication funded by the Max Planck Society. reasonable figure of merit involving power and efficiency, the optimal control strategy is to perform infinitesimal cycles around a fixed point. Furthermore, when the thermalization of the relevant quantities can be described by a single time-scale $\tau_{\text {eq }}$ (see details below), the optimal power output becomes proportional to $\mathcal{C} / \tau_{\text {eq }}$, where $\mathcal{C}$ is the heat capacity of the working substance (WS). Hence, the optimization of the heat engine cycle becomes intimately related to the maximization of $\mathcal{C}$ of the WS (interestingly, maximizing $\mathcal{C}$ is also crucial in thermometry [30-33]).

Then, we use these insights to design many-body heat engines that can operate at Carnot efficiency with finite power per constituent of the WS through a supraextensive scaling of $\mathcal{C} / \tau_{\text {eq }}$ (e.g., in a phase transition), in the spirit of [34,35] (see, also, [35-38]). Despite differences with respect to previous proposals, which were based on Otto engines [34,35], we find the same asymptotic scalings for performance, while proving by construction their optimality in the slow-driving regime. Other recent proposals towards the possibility of reaching Carnot efficiency at finite power have been developed in [34-36,39-45] (see, also, [15,46,47] for no-go results [48]).

Finite-time Carnot cycle.-We consider a quantum WS with a driven Hamiltonian $H(t)$, which interacts alternatively with a cold $\left(B_{c}\right)$ and a hot $\left(B_{h}\right)$ heat bath at temperature $T_{c}$ and $T_{h}$, respectively (the results presented in this Letter can be naturally extended to classical systems). The Carnot cycle consists of four steps: (i) While being coupled to $B_{c}, H(t)$ is modified continuously from $H(0)=H^{(X)}$ to $H\left(\tau_{c}^{-}\right)=H^{(Y)}$ for a time $\tau_{c}$. (ii) With the system isolated from the reservoirs, an adiabatic process is performed taking $H^{(Y)} \rightarrow H^{(Y)} T_{h} / T_{c}$. During this process $H(t)$ satisfies $\left[H(t), H\left(t^{\prime}\right)\right]=0 \forall t, t^{\prime}$ 
and commutes with the initial state [a Gibbs state with respect to $H\left(\tau_{c}^{-}\right)$], so it is possible to perform it arbitrarily quickly without affecting the state (hence, corresponding to Hamiltonian quenches). (iii) While being coupled to $B_{h}, H(t)$ is modified back from $H\left(\tau_{c}^{+}\right)=H^{(Y)} T_{h} / T_{c}$ to $H\left(\tau_{c}+\tau_{h}\right)=$ $H^{(X)} T_{h} / T_{c}$ in a time $\tau_{h}$. (iv) Finally, a second quench is performed to restore $H^{(X)} T_{h} / T_{c} \rightarrow H^{(X)}$, closing the cycle.

It is convenient to introduce the adimensional Hamiltonian $G(t):=\beta H(t)$, where $\beta=1 / k_{B} T$ is the inverse temperature of the bath to which the WS is coupled (we shall set the Boltzmann constant $k_{B}$ equal to $k_{B}=1$ ). Note that the Carnot cycle becomes smooth with respect to $G(t)$, and in what follows, we take the driving protocol to be time-reversal symmetric, more precisely that $G\left(s \tau_{c}\right)=$ $G\left(\tau_{c}+\tau_{h}(1-s)\right)$ with $s \in[0,1]$. This property is always satisfied by optimal heat engines in the LD regime as long as the two baths have the same spectral density [14], which is the subject of interest of this work. By expressing $G(t)$ as

$$
G\left(s \tau_{c}\right)=\sum_{j} \lambda_{j}(s) X_{j}, \quad s \in[0,1]
$$

where $\lambda_{j}(s)$ are the control parameters and $X_{j}$ the conjugated forces, the cycle control can be characterized by $\tau_{c}$, $\tau_{h}$ and its shape $\vec{\lambda}(s)$ (notice that $\tau_{c} \neq \tau_{h}$ in general; the time-reversal symmetry is intended in the adimensional unit $s$ ). We will not explicitly write the dependence on $s$, which will be clear from the context, but will indicate with a dot the time derivative with respect to $s$, i.e., $\dot{G} \equiv(\partial / \partial s) G \equiv \tau_{x}(d / d t) G, x=(h, c)$.

Now, we assume the slow driving or low dissipation approximation, which is crucial in this work. That is, $(d / d t) G$ is finite but small, so that we can expand the relevant quantities and keep only leading terms (formally, the small parameter of the expansion is $\tau_{\mathrm{eq}} / \tau_{x}$, where $\tau_{\mathrm{eq}}$ is the relaxation time of the dynamics). In this regime, the state of the WS is always close to thermal equilibrium, and the heat exchanged during the isotherms [steps (i) and (iii)] can be divided as

$$
Q_{x}=T_{x} \Delta S_{x}-W_{x}^{\text {diss }}, \quad x=(h, c),
$$

where $\Delta S_{x}$ is the reversible contribution obtained in the quasistatic limit $\tau_{x} \rightarrow \infty$, which is given by $\Delta S \equiv \Delta S_{h}=$ $-\Delta S_{c}=S\left[\omega\left(\tau_{c}\right)\right]-S[\omega(0)]$ with $\omega(t)=e^{-G(t)} / \operatorname{Tr}\left(e^{-G(t)}\right)$ and where $S(\rho)$ is the Von Neumann entropy. The irreversible term $W_{x}^{\text {diss }}$ can be described in this regime by the so called thermodynamic length $[20-25,28]$

$$
W_{x}^{\text {diss }}=\frac{T_{x}}{\tau_{x}} \int_{0}^{1} \sum_{i j} \dot{\lambda}_{i} m_{i j} \dot{\lambda}_{j} d s \equiv T_{x} \frac{\Sigma_{x}}{\tau_{x}}
$$

where $m_{i j}$ is given, when the driven observables $\left\langle X_{j}\right\rangle$ relax to equilibrium with the same timescale $\tau_{\text {eq }}$, by [20-25,28]

$$
m_{i j}=\tau_{\mathrm{eq}} \frac{\partial^{2} \ln \mathcal{Z}}{\partial \lambda_{i} \partial \lambda_{j}},
$$

where $\mathcal{Z}=\operatorname{Tr}\left(e^{-G}\right)$ is the partition function. Given the time-reversal symmetry of the driving protocol, it follows that $\Sigma_{h}=\Sigma_{c}$ [symmetric low-dissipation (SLD) regime]. Importantly, while the results presented in the main text are based upon the standard thermodynamic metric (4), generalizations (including $\tau_{\mathrm{eq}}$ depending on the trajectory $[24,25]$, the possibility of having several relaxation timescales, general Lindbladian dynamics [24,28], and protocols in which $\Sigma_{h} \neq \Sigma_{c}$ ) are developed in the Supplemental Material (SM) [49].

Optimization of the cycle.-Now, we optimize the power (and efficiency) of the Carnot engine over $\tau_{c}, \tau_{h}$ and its shape $\lambda_{j}(s)$, which are all the possible degrees of freedom. This enables us to obtain a fundamental upper bound on the power in the slow-driving regime, as well as the corresponding optimal control.

The work extracted during a cycle is given by $W=Q_{h}+Q_{c}$, and the total time is $\tau=\tau_{c}+\tau_{h}$. Hence, the power reads $P=\left(Q_{h}+Q_{c}\right) / \tau$, and the efficiency $\eta=\left(Q_{h}+Q_{c}\right) / Q_{h}$. By substituting the expressions (2)(3) and appropriately setting $\tau_{c}$ and $\tau_{h}$, one can maximize the power of the engine $\left(\partial P / \partial \tau_{j}=0\right)$ obtaining $[3,71]$

$$
P^{(\max )}=\frac{(\Delta S)^{2}}{4 \Sigma}\left(\sqrt{T_{h}}-\sqrt{T_{c}}\right)^{2},
$$

and the corresponding efficiency at maximum power (EMP) is given by the Curzon-Ahlborn (CA) EMP, $\eta_{\mathrm{CA}}=$ $1-\sqrt{T_{c} / T_{h}}$ [72]. In the most general case, one might seek, in order to not sacrifice completely the efficiency optimization over the power, to maximize a hybrid figure of merit $[7-9,15]$. The maximum efficiency for any given power output of the engine has been derived in [9] (see, also, [7]). Analogously, we can express the best power for a given efficiency, fixed to be a fraction of the maximum one,

$$
\eta=\gamma \eta_{C}, \quad \gamma \leq 1,
$$

where $\eta_{C}=1-T_{c} / T_{h}$ is the Carnot efficiency. In the SLD regime, this leads to a maximum power (cf. SM [49]),

$$
P_{\gamma}^{(\max )}=\frac{(\Delta S)^{2}}{4 \Sigma} \frac{\left(T_{h}-T_{c}\right)^{2} \gamma(1-\gamma)}{\gamma T_{c}+(1-\gamma) T_{h}},
$$

obtained by setting $\tau_{c}=2 \Sigma T_{c} /\left[\Delta S\left(T_{h}-T_{c}\right)(1-\gamma)\right]$ and $\tau_{h}=\tau_{c}\left[T_{h} T_{c}^{-1}(1-\gamma)+\gamma\right]$. Essentially, by tuning $\gamma$ in $\tau_{c}$ and $\tau_{h}$, one can interpolate between a maximally powerful engine with power (5) at efficiency $\eta_{\mathrm{CA}}$, and a Carnot engine with maximal efficiency and null power.

At this point, we note a crucial observation: after the optimization of $P$ over $\tau_{c}$ and $\tau_{h}$, the remaining figure of 
merit is always $(\Delta S)^{2} / \Sigma$, independent of the value of $\gamma$. In fact, this is a property that can be argued to be general given any figure of merit combining power and efficiency [49]. Now, we show how to maximize it by an opportune use of a Cauchy-Schwarz inequality. First, using the formula for the derivative of an exponential [73]: $\partial e^{-G} / \partial \lambda_{j}=$ $-\int_{0}^{1} e^{-G} X_{j} e^{-(1-s) G}$, we can again express $\Sigma$ in (3) in the more compact form

$$
\Sigma=\tau_{\mathrm{eq}} \int_{0}^{1} d s \operatorname{cov}_{\omega}(\dot{G}, \dot{G}),
$$

where $\operatorname{cov}_{\omega}(A, B)$ is the Kubo-Mori-Boguliobov inner product: $\quad \operatorname{cov}_{\omega}(A, B)=\int_{0}^{1} d s \operatorname{Tr}\left\{A \omega^{1-s}[B-\operatorname{Tr}(\omega B)] \omega^{s}\right\}$. Next, we note that the Von Neumann entropy $S=$ $-\operatorname{Tr}[\omega \ln \omega]$ satisfies: $\dot{S}=-\operatorname{Tr}(\dot{\omega} \ln \omega)=-\operatorname{cov}_{\omega}(\ln \omega, \dot{G})=$ $\operatorname{cov}_{\omega}(G, \dot{G})$ where we again used the formula $\dot{\omega}=$ $-\int_{0}^{1} d x \omega^{1-x}[\dot{G}-\operatorname{Tr}(\omega \dot{G})] \omega^{x}$, as well as $\operatorname{Tr}(\dot{\omega})=0$ and $\operatorname{cov}_{\omega}(A, \mathbb{1})=0$. Hence, we can write the total change in entropy $\Delta S$ as

$$
\Delta S=-\int_{0}^{1} d s \operatorname{cov}_{\omega}(G, \dot{G}) .
$$

Crucially, both $\Delta S$ and $\Sigma$ can be expressed through an infinite-dimensional scalar product given by: $\langle A, B\rangle_{\omega}=$ $\int_{0}^{1} d s \operatorname{cov}_{\omega}(A, B)$, that depends on the path $\left\{\lambda_{j}(s)\right\}$. Using the Cauchy-Schwarz (CS) inequality $|\langle A, B\rangle|^{2} \leq$ $\langle A, A\rangle\langle B, B\rangle$, the ratio $(\Delta S)^{2} / \Sigma$ can then be bounded as

$$
\frac{(\Delta S)^{2}}{\Sigma} \leq \frac{1}{\tau_{\mathrm{eq}}} \int_{0}^{1} d s \operatorname{cov}_{\omega}(G, G) \equiv \frac{1}{\tau_{\mathrm{eq}}} \int_{0}^{1} d s \mathcal{C},
$$

where $\mathcal{C}$ is the heat capacity of the WS,

$$
\mathcal{C}=-\beta^{2} \frac{\partial^{2} \ln \mathcal{Z}}{\partial \beta^{2}}=\operatorname{Tr}\left(\omega G^{2}\right)-\operatorname{Tr}(\omega G)^{2},
$$

i.e., the variance of the adimensional Hamiltonian $G$ for its thermal state. The CS inequality is saturated for $\dot{G} \propto G$, which means that creating quantum coherence cannot favor the power output in the slow driving regime, in agreement with Refs. [74,75]. More importantly, it shows that optimal thermodynamic protocols take the simple form $G(t)=\lambda(t) G(0)$.

We can further maximize (10) by noting that $\int_{0}^{1} d s \mathcal{C} \leq \max _{s} \mathcal{C}$. To saturate this inequality in practice, one needs to consider cycles where the modulation of $G$ is small

$$
\begin{aligned}
G\left(\tau_{c}\right) & =G(0)(1+\epsilon) \quad \epsilon \ll 1, \\
G\left(\tau_{c}+\tau_{h}\right) & =G(0),
\end{aligned}
$$

around an optimal point $G(0)$ where $\mathcal{C} / \tau_{\text {eq }}$ is maximized [as long as $\epsilon$ is small enough so that $G\left(s \tau_{c}\right)$ with $s \in[0,1]$ does not change substantially along the cycle, the precise form of $G\left(s \tau_{c}\right)$ is not important; see the SM for examples of explicit cycles]. In this case, in the limit $\epsilon \rightarrow 0$ the maximal power (7) for a given efficiency $\gamma \eta_{C}$ is given by

$$
P_{\gamma}^{(\max )}=\frac{\mathcal{C}}{4 \tau_{\mathrm{eq}}} \frac{\left(T_{h}-T_{c}\right)^{2} \gamma(1-\gamma)}{\gamma T_{c}+(1-\gamma) T_{h}}
$$

where $\mathcal{C}$ is the heat capacity at $G(0)$. We stress that (13) has been obtained after maximizing $P$ (for a fixed $\eta$ ) over all degrees of freedom: $\tau_{c}, \tau_{h}$ and the protocol $\left\{\lambda_{j}(s)\right\}$. This result shows a fundamental link between maximal power of a finite-time Carnot cycle and the heat capacity of the WS.

The simplicity of (13) can be contrasted to exact optimizations of heat engines [45,76-79], where the full solution easily becomes too complex or not even computable with the size of the WS, and with other geometric optimizations which require solving geodesic equations to design optimal paths in the parameter space [24,26,28,29,80-82]. In our approach, from the point of view of optimization, all that is left to do in (13) is to maximise $\mathcal{C}$ over the control parameters $\left\{\lambda_{j}\right\}$ to identify the optimal working point $G(0)$ in (12). In Fig. 1, explicit results are reported for the value of maximum $\mathcal{C}$, for different paradigmatic levels of control on the same system of $N$ qubits.

Crucially, this approach can be generalized to any metric $m_{i j}$ in (3) describing dissipation: in the SM [49], we show that the optimal control problem is always reduced to infinitesimal cycles, and the optimal working point can be found by a scalar maximization problem. We work out examples of standard microscopical dynamics in open quantum systems as well: a qubit, a qutrit, or a harmonic

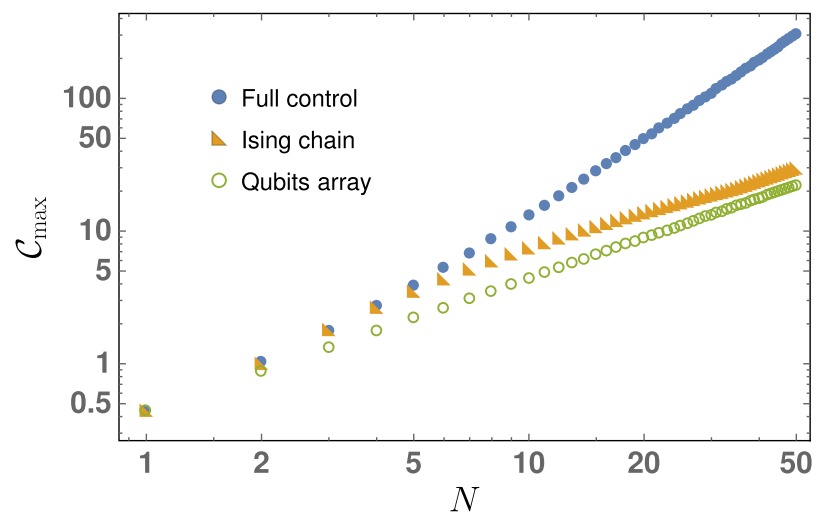

FIG. 1. Maximum adimensional $\mathcal{C}$ for a thermal system of $N$ qubits with different degrees of control [49]. (1) $C_{\max } \simeq 0.44 \mathrm{~N}$ for $N$ independent two-level systems with gap control. (2) For an Ising chain $H^{(N)}=-\lambda_{1}(t) \sum_{i=1}^{N} \sigma_{i}^{z} \sigma_{i+1}^{z}-\lambda_{2}(t) \sum_{i=1}^{N} \sigma_{i}^{z}$, we obtain $C_{\max } \simeq 0.59 N$. (3) Given complete control over the spectrum with $2^{N}$ levels, $C_{\max } \simeq N^{2} / 4$. Details can be found in [49]. 
oscillator as a WS in contact with bosonic thermal baths with different spectral densities.

It is important to point out that (13) should be understood as a theoretical ultimate upper bound on power, obtained by taking $\epsilon \rightarrow 0$ in (12). In practice, any experimental or realistic protocol will have finite $\epsilon$. In this case, as long as $\mathcal{C}$ is sufficiently smooth along the thermodynamic cycle (12), the power output $P$ [given by the integrated $\mathcal{C}$ in (10)] will not change considerably, so that realistic cycles will provide a more similar $P$ than the theoretically maximal one. In practice, keeping $\epsilon$ finite is also important to ensure the consistency of the slow-driving approximation $\tau_{\text {eq }} / \tau \ll 1$, given that, for the optimized protocols that lead to the power (7), one has $\tau \propto \epsilon$, more precisely

$$
\tau_{\mathrm{eq}} / \tau \sim \epsilon^{-1}\left(T_{h} / T_{c}-1\right)(1-\gamma) .
$$

Note that this can always be guaranteed for high efficiencies $\gamma \rightarrow 1$. From the same equation, we note, incidentally, that engines whose maximum efficiency is constrained to be low $\left(T_{c} / T_{h} \lesssim 1\right)$, i.e., arguably those engines that mostly need optimization in the high efficiency regime, show better convergence to the absolute bound.

Reaching Carnot efficiency at finite power.-As an example of application of the previous results, we now use the designed optimal finite-time Carnot cycles [Eqs. (12) where $G(0)$ will depend on each model of interest] to explore the possibility of reaching Carnot efficiency $\eta_{C}$ at finite power in the macroscopic limit. We follow the approach put forward in Refs. [34,35]: considering an $N$ particle WS, we aim at approaching Carnot efficiency in the macroscopic limit $N \rightarrow \infty$ without giving up power per constituent.

To reach Carnot efficiency, we need $\gamma=1$ in (6), and hence, we take $1-\gamma=N^{-\xi}$, where $\xi>0$ can be chosen at will. On the other hand, the maximal power $P_{\gamma}^{(\max )}$ in (13) depends only on $\mathcal{C}$ and $\tau_{\text {eq }}$; then, we assume $\mathcal{C}=c_{0} N^{1+a}$ and $\tau_{\text {eq }}=\tau_{0} N^{b}$, where the meaning of the different constants will be described for each model of interest later. Expanding the relevant quantities for $N \gg 1$, we obtain at leading order in $N$

$$
\begin{aligned}
P_{\gamma}^{(\max )} & =\frac{c_{0}\left(T_{c}-T_{h}\right)^{2}}{4 \tau_{0} T_{c}} N^{1+a-b-\xi}, \\
\tau_{c} & =\frac{2 \epsilon T_{c}}{T_{h}-T_{c}} \tau_{0} N^{b+\xi}, \quad \tau_{h}=\tau_{c}, \\
W & =\left(T_{h}-T_{c}\right) c_{0} \epsilon N^{1+a}, \quad \sigma_{w}^{2}=2\left(T_{h}-T_{c}\right) \frac{W}{\epsilon},
\end{aligned}
$$

where $\sigma_{w}^{2}=\left\langle w^{2}\right\rangle-(\langle w\rangle)^{2}$ is the variance of the work distribution, which measures the work fluctuations per cycle of the engine (see SM [49] for details on the calculation). Now, let us discuss two separate cases, inspired by [34] and [35], respectively.

(a) Control on the engine and the engine-bath interaction. First, we assume full control over the engine Hamiltonian with $2^{N}$ levels: that is, all levels can be modified at will by the experimentalist. While this is extremely challenging in practice, it is useful to obtain fundamental upper bounds on the maximal power. The optimal Hamiltonian maximizing $\mathcal{C}$ then consists in a ground level and a $2^{N}-1$ degenerate level (see $[30,83]$ ) which, as shown in Fig. 1, leads to $\mathcal{C} \propto N^{2}$, i.e., $a=1$. This supralinear scaling is obtained in an increasingly smaller region of the parameter space, which requires taking $\epsilon \propto 1 / N$ in (12) (see details in SM [49]), and also, this constrains from Eq. (14) $1-\gamma$ to scale accordingly, i.e., $\xi=1$. Furthermore, it is possible to reach, in realistic collisional scenarios, $\tau_{\text {eq }} \propto \sqrt{N}$ (i.e., $b=1 / 2$ ), or constant $\tau(b=0)$ if one is allowed to fine tune the interaction between the WS and the baths [34] [84]. in agreement with Ref. [34]. In the SM [49], we solve exactly this proposal for a feasible driving protocol close to the optimal one.

(b) Engine working on a phase transition point. A promising platform to obtain supralinear scaling of power with realistic control is by choosing the engine to work in a phase transition point of the many-body WS. For a finite WS operating close to the critical point, finite size scaling theory tells us that $\mathcal{C}$ develops a peak of height $C \propto$ $N^{1+\alpha /(\nu d)}$ and width $\delta \propto N^{-1 /(d \nu)}$, while $\tau_{\text {eq }} \propto N^{z / d}$ (here, $\alpha, \nu$, and $z$ correspond to the specific heat, correlation length, and dynamical critical exponents, while $d$ is the spatial dimension of the engine $[85,86])$. In order to exploit the critical scaling of the WS, we need to perform the cycle (12) where $\mathcal{C}$ becomes peaked, and hence, $\epsilon \propto \delta \propto N^{-1 /(d \nu)}$, which implies $\xi=1 /(d \nu)$ from Eq. (14). Then, from (15) with $a=\alpha /(\nu d)$ and $b=z / d$, supralinear scaling of $P_{\gamma}^{(\max )}$ is possible if

$$
\alpha-z \nu-1>0 .
$$

This condition is the same found for the Otto cycle proposed in [35]. Examples of physical systems where (16) is satisfied are also provided in [35], particularly in the presence of critical speed-ups of thermalization $(z<0$ [87-89]).

Besides efficiency and power, another crucial aspect of a heat engine is its reliability, i.e., the fluctuations in the output power. In fact, it has been recently pointed out in [36] that the Otto-cycle of [35] suffers from macroscopic fluctuations in the thermodynamic limit. For the Carnot cycle considered here, from (15), the relative work fluctuations read $f_{w}=\sigma_{w} / W=\sqrt{2\left(T_{h}-T_{c}\right) / \epsilon W}$. First of all, in case (a) where $a=1$ and $\epsilon \propto N^{-1}$ in (15), one has $f_{w} \sim \mathcal{O}(1)$ in the macroscopic limit. A similar situation takes place for the critical heat engine (b) as one 
simultaneously has $\epsilon \propto N^{-1 /(d \nu)}$ and $a=\alpha /(d \nu)$, and hence, $f_{w} \propto N^{(-2+d \nu+\alpha) /(d \nu)}$. Hence, using the relation $d \nu=2-\alpha$ [90], we obtain $f_{w} \sim \mathcal{O}(1)$. Therefore, for both proposals, $f_{w} \sim \mathcal{O}(1)$ in the thermodynamic limit, hence, hindering their reliability, which is the same result found in [36] for the Otto cycle. Despite $f_{w} \sim \mathcal{O}(1)$, these fluctuations can be suitably avoided when the number $M$ of cycles is large (the argument below applies to the Otto and Carnot cycle). Given $M$ cycles, we have that $f_{w} \propto 1 / \sqrt{M}$ as the average work $W \propto M$ whereas the work fluctuations $\sigma_{w} \propto$ $\sqrt{M}$ (think of a biased random walk). Therefore, the ratio between the fluctuations per unit time and the power goes to zero as $M$ grows even when the fluctuations per single cycle are large. Since we have that $M \propto \tau_{\text {tot }} / \tau_{\text {eq }}$, for a total time $\tau_{\text {tot }}$ of observation, fluctuations can be suitably avoided, e.g., for critical speed-ups where $\tau_{\mathrm{eq}} \propto N^{b}$ with $b<0$.

In actual implementations, the technical requirements to realize such optimized Carnot cycles are global control of the WS, $H(t)=\lambda(t) H(0)$, and enough precision to engineer small cycles in the region where $\mathcal{C} / \tau_{\text {eq }}$ has supralinear scaling with $N$. Since the width $\epsilon$ of this region shrinks with $N$, in a realistic implementation, the supralinear scaling will eventually be lost as the control precision is limited [50]. We remark that, even when the experimental control may be limited, our considerations provide upper bounds on the maximal power of finite-time Carnot engines.

Conclusions. - We have characterized the optimal cycle of a finite-time Carnot engine in the low-dissipation regime. The dissipation has been characterized by the thermodynamic metric (4), which is justified when the thermalization of the WS is well described by a single timescale $\tau_{\text {eq }}$. In this case, the optimal cycle turns out to be remarkably simple: it consists of modulations in the form $\lambda(t) H(0)$, where $H(0)$ is the Hamiltonian of the WS. The power output is then proportional to the heat capacity $\mathcal{C}$ of the WS, linking the optimal performance to the nature of the WS: as an application, we showed how the critical scaling of $\mathcal{C}$ can enable the design of optimal engines with extensive power reaching Carnot efficiency. These results have been generalized to general metrics in the SM [49], which we have used to derive the optimal cycle and corresponding power output of different WS (qubit, three-level system, or harmonic oscillator) interacting with a bosonic thermal bath. Putting everything together, our results provide a general framework to efficiently optimize the control of slowly driven Carnot engines.

We hope this work stimulates further investigations in the interplay between many-body physics and heat engines [35-38,51-57], as well as connections between performance, fluctuations, and degree of control [58]. In particular, our results hint at the answer for two open problems: (1) small cycles are optimal for engine performance in all regimes $[17,45,78]$, and (2) the performance of the proposals $[34,35]$ cannot be improved.

We thank Harry J. D. Miller and Karen Hovhannisyan for insightful discussions. We also thank the anonymous referees for constructive criticisms and useful feedback. P. A. is supported by "la Caixa" Foundation (ID 100010434, Grant No. LCF/BQ/DI19/11730023), the Spanish MINECO (Grant No. QIBEQI FIS2016-80773-P, and Severo Ochoa Grant No. SEV-2015-0522), Generalitat de Catalunya (Grant No. SGR1381 and CERCA Programme), Fundacio Privada Cellex.

*paolo.abiuso@icfo.eu

[1] S. Carnot, Réflexions sur la puissance motrice du feu et sur les machines propresa développer cette puissance (Bachelier, Paris, 1824).

[2] H. B. Callen, Thermodynamics and An Introduction to Thermostatistics (John Wiley \& Sons, New York, 1985), 2nd ed.

[3] M. Esposito, R. Kawai, K. Lindenberg, and C. Van den Broeck, Phys. Rev. Lett. 105, 150603 (2010).

[4] J. Guo, J. Wang, Y. Wang, and J. Chen, J. Appl. Phys. 113, 143510 (2013).

[5] C. V. den Broeck, Europhys. Lett. 101, 10006 (2013).

[6] A. C. Hernández, A. Medina, and J. M. M. Roco, New J. Phys. 17, 075011 (2015).

[7] V. Holubec and A. Ryabov, Phys. Rev. E 92, 052125 (2015).

[8] V. Holubec and A. Ryabov, J. Stat. Mech. (2016), 073204.

[9] Y.-H. Ma, D. Xu, H. Dong, and C.-P. Sun, Phys. Rev. E 98, 042112 (2018).

[10] C. de Tomás, A. C. Hernández, and J. M. M. Roco, Phys. Rev. E 85, 010104(R) (2012).

[11] C. de Tomas, J. M. M. Roco, A. C. Hernández, Y. Wang, and Z. C. Tu, Phys. Rev. E 87, 012105 (2013).

[12] Y. Wang, M. Li, Z. C. Tu, A. C. Hernández, and J. M. M. Roco, Phys. Rev. E 86, 011127 (2012).

[13] Y. Hu, F. Wu, Y. Ma, J. He, J. Wang, A. C. Hernandez, and J. M. M. Roco, Phys. Rev. E 88, 062115 (2013).

[14] V. Cavina, A. Mari, and V. Giovannetti, Phys. Rev. Lett. 119, 050601 (2017).

[15] N. Shiraishi, K. Saito, and H. Tasaki, Phys. Rev. Lett. 117, 190601 (2016).

[16] M. Esposito, R. Kawai, K. Lindenberg, and C. Van den Broeck, Phys. Rev. E 81, 041106 (2010).

[17] P. Abiuso and V. Giovannetti, Phys. Rev. A 99, 052106 (2019).

[18] F. Weinhold, J. Chem. Phys. 63, 2479 (1975).

[19] G. Ruppeiner, Phys. Rev. A 20, 1608 (1979).

[20] P. Salamon and R. S. Berry, Phys. Rev. Lett. 51, 1127 (1983).

[21] F. Schlögl, Z. Phys. B 59, 449 (1985).

[22] J. Nulton, P. Salamon, B. Andresen, and Q. Anmin, J. Chem. Phys. 83, 334 (1985).

[23] G. E. Crooks, Phys. Rev. Lett. 99, 100602 (2007).

[24] D. A. Sivak and G. E. Crooks, Phys. Rev. Lett 108, 190602 (2012). 
[25] M. V. S. Bonança and S. Deffner, J. Chem. Phys. 140, 244119 (2014).

[26] P. R. Zulkowski and M. R. DeWeese, Phys. Rev. E 92 , 032113 (2015).

[27] D. Mandal and C. Jarzynski, J. Stat. Mech. (2016) 063204.

[28] M. Scandi and M. Perarnau-Llobet, Quantum 3, 197 (2019).

[29] H. J. D. Miller, M. Scandi, J. Anders, and M. PerarnauLlobet, Phys. Rev. Lett. 123, 230603 (2019).

[30] L. A. Correa, M. Mehboudi, G. Adesso, and A. Sanpera, Phys. Rev. Lett. 114, 220405 (2015).

[31] P. P. Potts, J. B. Brask, and N. Brunner, Quantum 3, 161 (2019).

[32] K. V. Hovhannisyan and L. A. Correa, Phys. Rev. B 98, 045101 (2018).

[33] M. Mehboudi, A. Sanpera, and L. A. Correa, J. Phys. A 52, 303001 (2019).

[34] A. E. Allahverdyan, K. V. Hovhannisyan, A. V. Melkikh, and S. G. Gevorkian, Phys. Rev. Lett. 111, 050601 (2013).

[35] M. Campisi and R. Fazio, Nat. Commun. 7, 11895 (2016).

[36] V. Holubec and A. Ryabov, Phys. Rev. E 96, 030102(R) (2017).

[37] Y.-H. Ma, S.-H. Su, and C.-P. Sun, Phys. Rev. E 96, 022143 (2017).

[38] S. Chand and A. Biswas, Phys. Rev. E 98, 052147 (2018).

[39] M. Mintchev, L. Santoni, and P. Sorba, arXiv:1310.2392.

[40] M. Polettini, G. Verley, and M. Esposito, Phys. Rev. Lett. 114, 050601 (2015).

[41] J. Koning and J. O. Indekeu, Eur. Phys. J. B 89, 248 (2016).

[42] M. Polettini and M. Esposito, Europhys. Lett. 118, 40003 (2017).

[43] C. V. Johnson, Phys. Rev. D 98, 026008 (2018).

[44] V. Holubec and A. Ryabov, Phys. Rev. Lett. 121, 120601 (2018).

[45] P. A. Erdman, V. Cavina, R. Fazio, F. Taddei, and V. Giovannetti, New J. Phys. 21, 103049 (2019).

[46] N. Shiraishi, Phys. Rev. E 92, 050101(R) (2015).

[47] N. Shiraishi and H. Tajima, Phys. Rev. E 96, 022138 (2017).

[48] Note that these results are not in contradiction with the present analysis, as we are concerned with reaching Carnot efficiency asymptotically.

[49] P. Abiuso and M. Perarnau-Llobet; See Supplemental material at http://link.aps.org/supplemental/10.1103/ PhysRevLett.124.110606 for optimal cycles for lowdissipation heat engines, which includes Refs. [50-70].

[50] Other possible implementation-dependent limitations may be the cost of turning on or off the interaction with the baths $[52,91]$, and a nonzero time for the quenches.

[51] E. Mascarenhas, H. Bragança, R. Dorner, M. F. Santos, V. Vedral, K. Modi, and J. Goold, Phys. Rev. E 89, 062103 (2014).

[52] M. Perarnau-Llobet, A. Riera, R. Gallego, H. Wilming, and J. Eisert, New J. Phys. 18, 123035 (2016).

[53] J. Lekscha, H. Wilming, J. Eisert, and R. Gallego, Phys. Rev. E 97, 022142 (2018).

[54] N. Y. Halpern, C. D. White, S. Gopalakrishnan, and G. Refael, Phys. Rev. B 99, 024203 (2019).

[55] M. Kloc, P. Cejnar, and G. Schaller, Phys. Rev. E 100, 042126 (2019).

[56] J. Jaramillo, M. Beau, and A. del Campo, New J. Phys. 18, 075019 (2016).
[57] W. Niedenzu and G. Kurizki, New J. Phys. 20, 113038 (2018).

[58] M. Bauer, K. Brandner, and U. Seifert, Phys. Rev. E 93, 042112 (2016).

[59] R. Alicki, J. Phys. A 12, L103 (1979).

[60] J. Anders and V. Giovannetti, New J. Phys. 15, 033022 (2013).

[61] T. D. Kieu, Phys. Rev. Lett. 93, 140403 (2004).

[62] S. Vinjanampathy and J. Anders, Contemp. Phys. 57, 545 (2016).

[63] M. Campisi, S. Denisov, and P. Hänggi, Phys. Rev. A 86, 032114 (2012).

[64] T. V. Acconcia, M. V. S. Bonança, and S. Deffner, Phys. Rev. E 92, 042148 (2015).

[65] M. F. Ludovico, F. Battista, F. von Oppen, and L. Arrachea, Phys. Rev. B 93, 075136 (2016).

[66] H.-P. Breuer, F. Petruccione et al., The Theory of Open Quantum Systems (Oxford University Press on Demand, Oxford, 2002).

[67] T. Albash, S. Boixo, and D. A. Lidar, New J. Phys. 14, 123016 (2012).

[68] R. Dann, A. Levy, and R. Kosloff, Phys. Rev. A 98, 052129 (2018).

[69] M. Yamaguchi, T. Yuge, and T. Ogawa, Phys. Rev. E 95, 012136 (2017).

[70] H. J. D. Miller et al. (to be published).

[71] T. Schmiedl and U. Seifert, Europhys. Lett. 81, 20003 (2007).

[72] F. L. Curzon and B. Ahlborn, Am. J. Phys. 43, 22 (1975).

[73] F. Hiai and D. Petz, Introduction to Matrix Analysis and Applications (Springer International Publishing, New York, 2014), pp. 101-135.

[74] K. Brandner, M. Bauer, and U. Seifert, Phys. Rev. Lett. 119, 170602 (2017).

[75] Y.-H. Ma, D. Xu, H. Dong, and C.-P. Sun, Phys. Rev. E 98 , 022133 (2018).

[76] T. Schmiedl and U. Seifert, Phys. Rev. Lett. 98, 108301 (2007).

[77] A. E. Allahverdyan, K. Hovhannisyan, and G. Mahler, Phys. Rev. E 81, 051129 (2010).

[78] V. Cavina, A. Mari, A. Carlini, and V. Giovannetti, Phys. Rev. A 98, 012139 (2018).

[79] P. Menczel, T. Pyhäranta, C. Flindt, and K. Brandner, Phys. Rev. B 99, 224306 (2019).

[80] P. R. Zulkowski, D. A. Sivak, and M. R. DeWeese, PLoS One 8, e82754 (2013).

[81] G. M. Rotskoff and G. E. Crooks, Phys. Rev. E 92, 060102 (R) (2015).

[82] G. M. Rotskoff, G. E. Crooks, and E. Vanden-Eijnden, Phys. Rev. E 95, 012148 (2017).

[83] D. Reeb and M. M. Wolf, New J. Phys. 16, 103011 (2014).

[84] We note that, because of the degeneracies in the Hamiltonian of the WS, the steady state might not be unique (as the population in each degenerate energy state might depend on the initial state). However, this is irrelevant as we are considering a driving $\dot{G} \propto G$, where the power output depends only on the total population in each degenerate energy level. 
[85] M. E. Fisher and M. N. Barber, Phys. Rev. Lett. 28, 1516 (1972).

[86] M. Suzuki, Prog. Theor. Phys. 58, 1142 (1977).

[87] H. Boukari, M.E. Briggs, J. N. Shaumeyer, and R. W. Gammon, Phys. Rev. Lett. 65, 2654 (1990).

[88] C. P. Grams, M. Valldor, M. Garst, and J. Hemberger, Nat. Commun. 5, 4853 (2014).
[89] M. Tavora, A. Rosch, and A. Mitra, Phys. Rev. Lett. 113, 010601 (2014).

[90] K. Huang, Introduction to Statistical Physics (Chapman and Hall/CRC, Boca Raton, 2009).

[91] D. Newman, F. Mintert, and A. Nazir, Phys. Rev. E 95, 032139 (2017). 